\title{
How to improve patient selection for neoadjuvant chemotherapy in bladder cancer patients candidate for radical cystectomy and pelvic lymph node dissection
}

\author{
Stefania Zamboni ${ }^{1,2} \cdot$ Marco Moschini $^{1,3} \cdot$ Alessandro Antonelli $^{2} \cdot$ Claudio Simeone $^{2} \cdot$ Sandra Belotti $^{2}$. \\ Luca Cristinelli ${ }^{2} \cdot$ Francesco Montorsi $^{3} \cdot$ Alberto Briganti $^{3} \cdot$ Andrea Gallina $^{3} \cdot$ Andrea Salonia $^{3} \cdot$ Renzo Colombo $^{3}$. \\ Livio Mordasini ${ }^{1} \cdot$ Agostino Mattei $^{1} \cdot$ Philipp Baumeister $^{1}$
}

Received: 6 February 2019 / Accepted: 20 August 2019

๑) Springer-Verlag GmbH Germany, part of Springer Nature 2019

\begin{abstract}
Purpose To improve patient selection for neoadjuvant chemotherapy (NAC) before radical cystectomy (RC) in bladder cancer patients $(\mathrm{BCa})$.

Methods Retrospective evaluation of 1057 patients with cT2-4N0M0 BCa treated with RC and pelvic lymph node dissection between 1990 and 2018 at 3 referral centers. Adverse pathologic features (APF) were defined as pT3-pT4/pN + disease at RC. A regression tree model (CART) was used to assess preoperative risk group classes. A multivariable logistic regression (MVA) was performed to identify predictors of APF at RC.

Results Median age was 70 years and most of the patients were men (83\%). Of the 1057 patients included in our study, 688 $(65 \%)$ had APF. CART analysis was able to stratify patients into 3 risk groups: low (cT2 and single disease, odds ratio [OR] 0.62), intermediate (cT2 and multiple disease, OR 1.08), and high (cT3-cT4, OR 1.28). On MVA APF were associated with variant histology (odds ratio [OR] 3.97, 95\% confidence interval [CI] 1.46-10.83, $p=0.007$ ), multifocality at TUR (OR 2.56, CI 1.27-5.17, $p=0.09$ ), completeness of resection (OR 0.47, CI $0.23-0.96, p=0.04$ ) and clinical extravesical disease (OR 3.42, CI 1.63-7.14, $p=0.001$ ).

Conclusion We defined three pre-operative risk classes. Our results indicate that patients with a cT3-T4 disease are those who might benefit more from NAC whereas those with T2 single disease should be those to whom NAC probably shouldn't be proposed. Given the high rate of understaging in BCa patients, NAC can be proposed in selected cases of cT2/multifocal disease.
\end{abstract}

Keywords Bladder cancer $\cdot$ Radical cystectomy $\cdot$ Neoadjuvant chemotherapy

\section{Introduction}

Bladder cancer (BCa) is the second most common genitourinary malignancy with 81,190 estimated new diagnosis in 2018 in the United States only [1]. About 25\% of patients

Stefania Zamboni

stefania.zamboni@libero.it

1 Klinik für Urologie, Luzerner Kantonsspital, Lucerne, Switzerland

2 Spedali Civili Hospital of Brescia, University of Brescia, Brescia, Italy

3 Unit of Urology/Division of Oncology, IRCCS Ospedale San Raffaele, URI, Milan, Italy with $\mathrm{BCa}$, presents at first diagnosis a muscle-invasive disease, whose gold standard treatment is radical cystectomy (RC) with bilateral pelvic lymph node dissection (PLND) [2]; however, $\mathrm{RC}$ alone, provides an overall 5-year survival in approximately half of the patients, probably as a result of the presence of radiologically non-detectable micrometastasis. Cisplatinum-based neoadjuvant chemotherapy (NAC) confers an overall survival benefit of $8 \%$ at 5 years [3] in patients with a cT2-T4a non-metastatic disease and its use is recommended by international guidelines [2, 4]. Unfortunately, although there is a documented benefit in survival, this treatment is underutilized worldwide with some series reporting that from 1.4 to $20.9 \%$ only of eligible patients are effectively treated with NAC [5-8]. Several reasons are probably the cause of this under-use such as 
numerous patients' comorbidities, low performance status, poor renal function [9], the concern of delay of RC [10] and of an increase in surgical morbidities, even if Grossman et al. [11] reported no difference between patients treated with RC alone and with RC preceded by NAC. For all these reasons, an accurate selection of patients who can benefit more from NAC is very important. Therefore, the aim of our study was to analyze a cohort of patients treated with $\mathrm{RC}$ alone, defining preoperative risk-groups to help urologists to identify best candidates for NAC.

\section{Materials and methods}

We retrospectively investigated 1057 patients who underwent RC without NAC for clinical T2-4N0 non-metastatic BCa between 1990 and 2018 at three tertiary referral centers. All data regarding demographics characteristics, last transurethral resection (TUR) before RC and RC characteristics were collected in prospectively maintained institutional databases. Patients with a suspicion of $\mathrm{BCa}$ were valuated with a TUR and staged preoperatively with pelvic/abdominal computerized tomography $(\mathrm{CT})$, bone scan when indicated and chest X-ray or thoracic CT scan. Dedicated pathologists examined all TUR and RC specimens: tumor grade was evaluated according 1973 WHO grading system for all patients who underwent TUR or RC between 2001 and 2004 and, for patients submitted to surgery later, according grading WHO 2004 [12]. Concomitant carcinoma in situ (CIS) was defined as the presence of CIS at TUR specimens in association with another pathological stage and variant histology any presence of urothelial or nonurothelial variants at the pathological report [13]. Multifocality was visually evaluated and defined as the presence of more than one macroscopic disease; complete resection was visually evaluated and defined as the absence of macroscopic residual tumor at the end of the TUR.

Adverse pathological features (APF) were defined as the presence of a pT3-pT4 stage and/or positive pathologic node stage disease at $\mathrm{RC}$ which correspond to the preoperative inclusion criteria for adjuvant chemotherapy. Bladder cancer staging was assessed according to VI edition TNM classification [14]. Follow-up consisted of a visit every 3-4 months for the first year after surgery, biannual during the second year and annual successively: examinations included radiological imaging with a chestabdominal CT scan in all patients. In addition to physical examination laboratory testing, intravenous pyelography, endoscopic view of the neobladder, urine cytology, urethral washings and bone scan were carried out if indicated.

\section{Primary and secondary endpoint}

Primary endpoint was to assess pre-operative risk classes to find which categories of patients can benefit more from NAC before RC. Secondary endpoint was to assess pre-operative predictors of APF after RC.

\section{Statistical analyses}

Descriptive statistics of categorical variables focused on frequencies and proportions. Means, medians, and interquartile ranges (IQR) were reported for continuously coded variables. The Kruskal-Wallis test and Chi square test were used to compare the statistical significance of differences in medians and proportions, respectively. Univariable and multivariable logistic regression analyses were used to assess predictors of APF. Multivariable models were adjusted for all available preoperative features: age, gender, presence of pre-operative hydronephrosis, concomitant CIS at TUR, presence of histological variants, multifocality, completeness of TUR, clinical T stage and clinical nodal stage. A regression tree model (CART) was used to assess preoperative risk group classes. Statistical significance was considered at $p<0.05$. Statistical analyses were performed using STATA $14.0^{\circledR}$ (Stata Corp., College Station, TX, USA).

\section{Results}

We evaluated 1057 patients with clinical non-metastatic $\mathrm{BCa}$ treated with RC between 1990 and 2018. Baseline pre-operative characteristic are reported in Table 1. Median age was $70(63-76)$ years and most of the patients were men $(n=874$, $83 \%)$. At post-operative evaluation 688 (65\%) had APF.

A decision tree generated by CART analyses was tested for its ability to stratify patients according to risk of presenting APF. This tree was able to stratify patients into 3 risk groups: low (cT2 and single disease, OR 0.62), intermediate (cT2 and multiple disease, OR 1.08), and high (cT3-cT4, OR 1.28) (Fig. 1). Univariable and multivariable logistic regression analyses predicting APF are reported in Table 2. On univariable analyses age (odds ratio [OR] 1.01, 95\% confidence interval $[\mathrm{CI}] 1.00-1.02, p=0.02)$, presence of hydronephrosis (OR 2.29, CI 1.49-3.53, $p<0.001$ ), concomitant CIS at TUR (OR 0.45, CI 0.29-0.71, $p=0.001$, multifocality at TUR (OR 1.50, CI 1.07-2.09, $p=0.01$ ), completeness of resection (OR 0.62, CI 0.43-0.90, $p=0.01$ ), cT3-4 vs cT $=2$ (OR 1.92, CI 1.27-2.90, $p=0.02$ ) were associated with an increased risk of APF at RC.

On multivariable logistic regression analyses APF were associated with variant histology at TUR (OR 3.97, CI 
Table 1 Baseline pre-operative characteristic of patients treated with radical cystectomy ( $\mathrm{RC}$ ) and pelvic lymph node dissection for bladder cancer (BCa) stratified according the presence of adverse pathologic features (AFP, defined as the presence of a pT3-pT4 stage and/ or positive pathologic node stage disease at $\mathrm{RC}$ and/or the occurrence of an early recurrence after RC)

\begin{tabular}{|c|c|c|c|c|}
\hline Variables & $\begin{array}{l}\text { Overall } \\
(n=1057, \\
100 \%)\end{array}$ & $\begin{array}{l}\text { No APF } \\
(n=364, \\
35 \%)\end{array}$ & $\begin{array}{l}\text { APF }(n=688, \\
65 \%)\end{array}$ & $p$ value \\
\hline \multicolumn{5}{|l|}{ Age, years } \\
\hline Mean & 69 & 68 & 69 & \multirow[t]{2}{*}{0.01} \\
\hline $\begin{array}{r}\text { Median } \\
(\mathrm{IQR})\end{array}$ & $70(63-76)$ & $69(62-75)$ & $70(63-77)$ & \\
\hline \multicolumn{5}{|l|}{ Gender } \\
\hline Male & $874(83 \%)$ & $309(85 \%)$ & $561(82 \%)$ & \multirow[t]{2}{*}{0.2} \\
\hline Female & $182(17 \%)$ & $55(15 \%)$ & $127(18 \%)$ & \\
\hline \multicolumn{5}{|c|}{ Hydronephrosis } \\
\hline No & $352(74 \%)$ & $186(82 \%)$ & $166(67 \%)$ & \multirow[t]{2}{*}{$<0.001$} \\
\hline Yes & $124(26 \%)$ & $40(18 \%)$ & $82(33 \%)$ & \\
\hline \multicolumn{5}{|c|}{ CIS at TUR } \\
\hline No & $841(91 \%)$ & $272(86 \%)$ & $566(93 \%)$ & \multirow[t]{2}{*}{$<0.001$} \\
\hline Yes & $88(9.0 \%)$ & $45(14 \%)$ & $43(7.0 \%)$ & \\
\hline \multicolumn{5}{|c|}{ Variant histology at TUR } \\
\hline No & $630(86 \%)$ & $234(89 \%)$ & $395(84 \%)$ & \multirow[t]{2}{*}{0.06} \\
\hline Yes & $103(14 \%)$ & $28(11 \%)$ & $74(16 \%)$ & \\
\hline \multicolumn{5}{|c|}{ Unifocality at TUR } \\
\hline No & $282(47 \%)$ & $95(41 \%)$ & $186(51 \%)$ & \multirow[t]{2}{*}{0.01} \\
\hline Yes & $314(53 \%)$ & $136(59 \%)$ & $177(49 \%)$ & \\
\hline \multicolumn{5}{|c|}{ Complete TUR } \\
\hline No & $304(60 \%)$ & $110(54 \%)$ & $194(65 \%)$ & \multirow[t]{2}{*}{0.01} \\
\hline Yes & $201(40 \%)$ & $95(46 \%)$ & $105(35 \%)$ & \\
\hline \multicolumn{5}{|l|}{ cT stage } \\
\hline $\mathrm{cT} 2$ & $312(64 \%)$ & $125(74 \%)$ & $187(60 \%)$ & \multirow[t]{2}{*}{0.002} \\
\hline cT3-4 & $173(36 \%)$ & $44(26 \%)$ & $127(40 \%)$ & \\
\hline
\end{tabular}

$A P F$ adverse pathological features, IQR interquartile range, $C I S$ carcinoma in situ, TUR transurethral resection of bladder
$1.46-10.83, p=0.007)$, multifocality at TUR (OR 2.56, CI $1.27-5.17, p=0.009$ ), completeness of resection (OR 0.47 , CI $0.23-0.96, p=0.04)$ and cT3 -4 vs $\mathrm{cT}=2$ (OR 3.42, CI $1.63-7.14, p=0.001)$.

\section{Discussion}

Although the administration of NAC is recommended in patients with $\mathrm{cT} 2-\mathrm{T} 4 \mathrm{BCa}$, this treatment is underutilized worldwide, especially in Europe [8]. Moreover, the role of NAC in patients with $\mathrm{T} 2$ diseases remains controversial, since these patients have a 5-year survival of approximately $80 \%$ when treated with RC alone [15]. This consideration could lead to an "a priori" decision to exclude all patients with a clinical T2 diseases from NAC, even if it has been largely demonstrated that current techniques of staging are inaccurate for BCa-patients, with some series reporting more than $40 \%$ of understaging $[16,17]$. That would then translate in the omission of NAC in patients classified as "T2", who are found to have an extravesical disease at RC specimen and consequently, would have probably benefit from NAC. The presence of APF and in particular, extravesical disease and positive nodes at RC specimens, has been reported associated with worse survival outcomes and, therefore, patients with these pathological characteristics are those who might benefit more from chemotherapy pre-surgery. Considering all these issues our main objective was to detect pre-surgical characteristics associated with APF to improve selection for NAC. To make this possible, we applied the CART regression tree analysis, which is a statistical approach able to detect interactions between variables similarly to multivariable logistic regression analysis, but additionally is able to build a decision tree.

We made several findings: first and foremost, in our study the CART method identified two pre-operative variables
Fig. 1 Decision tree generated by CART analyses, stratifying patients with clinical nonmetastatic bladder cancer $(\mathrm{BCa})$ treated with radical cystectomy (RC) and pelvic lymph node dissection (PLND) according to risk of presenting APF after RC

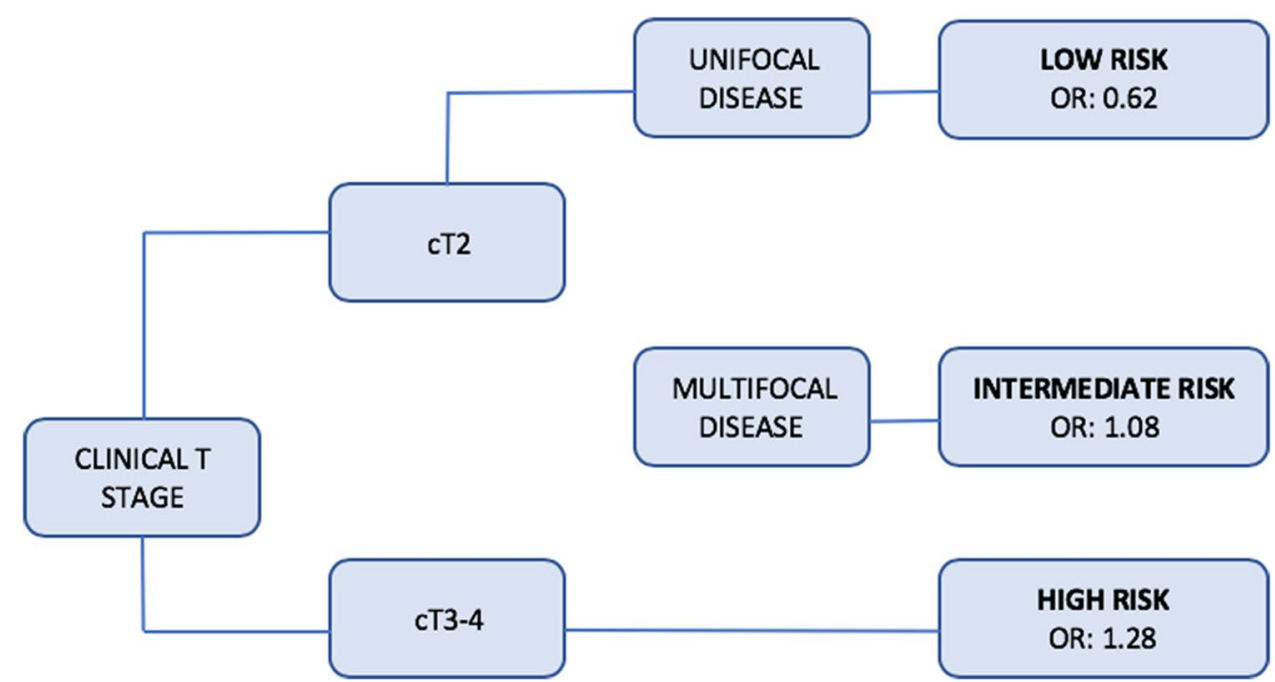


Table 2 Univariable and multivariable logistic regression analyses predicting adverse pathological feature (APF) after radical cystectomy $(\mathrm{RC})$ and pelvic lymph node dissection

\begin{tabular}{|c|c|c|c|c|}
\hline \multirow[t]{2}{*}{ Variables } & \multicolumn{2}{|c|}{ Univariable analyses } & \multicolumn{2}{|c|}{ Multivariable analyses } \\
\hline & OR $(95 \% \mathrm{CI})$ & $p$ value & OR $(95 \% \mathrm{CI})$ & $p$ value \\
\hline Age, years & $1.01(1.00-1.02)$ & 0.02 & $1.02(0.99-1.07)$ & 0.1 \\
\hline \multicolumn{5}{|l|}{ Gender } \\
\hline Male & Ref & Ref & Ref & Ref \\
\hline Female & $0.78(0.55-1.11)$ & 0.2 & $1.01(0.42-2.42)$ & 0.9 \\
\hline Hydronephrosis yes vs. no & $2.29(1.49-3.53)$ & $<0.001$ & $1.42(0.66-3.07)$ & 0.4 \\
\hline CIS at TUR yes vs. no & $0.45(0.29-0.71)$ & 0.001 & $0.51(0.18-1.43)$ & 0.2 \\
\hline Variant histology yes vs. no & $1.56(0.98-2.48)$ & 0.06 & $3.97(1.46-10.83)$ & 0.007 \\
\hline Multifocality yes vs. no & $1.50(1.07-2.09)$ & 0.01 & $2.56(1.27-5.17)$ & 0.009 \\
\hline Complete TUR yes vs. no & $0.62(0.43-0.90)$ & 0.01 & $0.47(0.23-0.96)$ & 0.04 \\
\hline \multicolumn{5}{|l|}{ cT stage } \\
\hline $\mathrm{cT}=2$ & Ref & Ref & Ref & Ref \\
\hline cT3-4 & $1.92(1.27-2.90)$ & 0.002 & $3.42(1.63-7.14)$ & 0.001 \\
\hline
\end{tabular}

$O R$ odds ratio, $C I$ confidence interval, $C I S$ carcinoma in situ, TUR transurethral resection (clinical T stage and number of tumors) as significant predictors of APF. These two variables permitted a stratification of patients in 3 risks groups according ORs: the low-risk group includes patients with a clinical T2 unifocal disease who are probably those who don't benefit from NAC and to whom NAC shouldn't be proposed; high-risk group includes patients with clinical T3-4 stage (unifocal or multifocal) and are those who can benefit more from NAC and to whom NAC should be always proposed; lastly, the intermediaterisk group which includes patients with a clinical T2 multifocal disease who can probably benefit from NAC, but less compared to the high-risk group and to whom NAC should be proposed in selected cases. In particular, since NAC is recommended in all guidelines $[2,4]$ in T2-T4a non-metastatic disease, our finding can improve decision-making in patients with clinical T2 diseases which, as previously mentioned, are one of the categories in which administration of NAC remains controversial.

Clinical T staging, but not organ confined disease, was also a predictor of worse survival in Culp et al. study [18, 19]: risk groups were found according to survival analyses in patients who underwent NAC and RC and the high-risk group included patients with pre-operative hydroureteronephrosis, cT3b-T4a disease, and/or histological evidence of lymphovascular invasion, micropapillary or neuroendocrine features at transurethral resection. Even if Culp et al. study [18] reported an association between histological variants and worse survival outcomes, in our study the CART analysis didn't include it in the decision tree. Anyway, in our study variant histology at TUR was associated to APF after $\mathrm{RC}$ at multivariable logistic regression analyses $(p=0.007)$. Our results are in accordance with Abufaraj et al. [20], who reported a significant relationship $(p=0.04)$ between variant histology at TUR and APF (such as lymph node metastasis and advanced pathological $\mathrm{T}$ stage). All these findings support results reported in several previous studies, which have underlined the importance of variant histology as prognostic factor in patients affected by $\mathrm{BCa}$ in term of higher risk of APF, worse survival outcomes [21] and also higher risk of progression in patients with non-muscle invasive bladder disease [22] compared to patients affected by standard urothelial histology.

Our study is not without limitation. First of all, our study is limited by its retrospective design and inherent biases. While data were recorded prospectively and were adequately transferred to institutional databases, all the analyses were retrospective. Second, in the present cohort, clinical evaluation was performed by different radiologist and we did not perform a central review of all CT scans. This aspect might have impact on the results of clinical $\mathrm{T}$ stage. Same was for all pathological TUR and RC specimens, which have not been submitted to a central review although were all analyzed by dedicated uro-pathologists. Third, all patients included in our cohort underwent TUR and RC at highvolume referral centers, therefore our findings might not be applicable to other non-referral centers. Fourth, data regarding presence of lymphovascular, which is a well-known prognostic factors for lymph nodes invasion and upstaging, and data regarding presence of variant histology were not available in our dataset.

\section{Conclusion}

We found three pre-operative risk classes to help urologist to identify patients who can benefit more from NAC before RC. Our results suggest that patients with a clinical T3-T4 disease are those who might benefit more from NAC whereas patients with a cT2 unifocal disease might be those who don't benefit from this treatment. Moreover, given 
the high rate of understaging in $\mathrm{BCa}$ patients and the result of our regression tree, we suggest to propose NAC also in selected cases of clinical cT2 disease with multifocal BCa (intermediate group). Nonetheless, more studies are needed to confirm our results in order to facilitate patient selection for NAC.

Author contributions SZ: manuscript writing, statistical analyses, data collection; MM: project development, data collection, drafting of the manuscript; AA: critical revision of the manuscript; CS: critical revision of the manuscript; SB: drafting of the manuscript; LC: drafting of the manuscript; FM: critical revision of the manuscript; AB: critical revision of the manuscript; AG: critical revision of the manuscript; AS: critical revision of the manuscript; LM: critical revision of the manuscript; AM: critical revision of the manuscript; PB: project development, supervision, critical revision of the manuscript.

\section{Compliance with ethical standards}

Conflict of interest The authors declare that they have no conflict of interest.

Ethical standards All person gave their informed consent to use their data for this retrospective study.

Ethical approval All procedures performed in studies involving human participants were in accordance with the ethical standards of the institutional and/or national research committee and with the 1964 Helsinki declaration and its later amendments or comparable ethical standards.

\section{References}

1. Siegel RL, Miller KD, Jemal A (2018) Cancer statistics, 2018. CA Cancer J Clin 68:7-30. https://doi.org/10.3322/caac.21442

2. Alfred Witjes J, Lebret T, Compérat EM et al (2017) Updated 2016 EAU guidelines on muscle-invasive and metastatic bladder cancer. Eur Urol 71:462-475. https://doi.org/10.1016/j.eurur o.2016.06.020

3. Stein JP (2003) Contemporary concepts of radical cystectomy and the treatment of bladder cancer. J Urol 169:116-117. https://doi. org/10.1097/01.ju.0000042054.84500.10

4. Chang SS, Bochner BH, Chou R et al (2017) Treatment of nonmetastatic muscle-invasive bladder cancer: AUA/ASCO/ASTRO/ SUO guideline. J Urol 198:552-559. https://doi.org/10.1016/j. juro.2017.04.086

5. Anan G, Hatakeyama S, Fujita N et al (2017) Trends in neoadjuvant chemotherapy use and oncological outcomes for muscleinvasive bladder cancer in Japan: a multicenter study. Oncotarget 8:86130-86142

6. Duplisea JJ, Mason RJ, Reichard CA et al (2018) Trends and disparities in the use of neoadjuvant chemotherapy for muscleinvasive urothelial carcinoma. Can Urol Assoc J. https://doi. org/10.5489/cuaj.5405

7. Martini T, Gilfrich C, Mayr R et al (2017) The use of neoadjuvant chemotherapy in patients with urothelial carcinoma of the bladder: current practice Among clinicians. Clin Genitourin Cancer 15:356-362. https://doi.org/10.1016/j.clgc.2016.09.003

8. Reardon ZD, Patel SG, Zaid HB et al (2015) Trends in the use of perioperative chemotherapy for localized and locally advanced muscle-invasive bladder cancer: a sign of changing tides. Eur Urol 67:165-170. https://doi.org/10.1016/j.eururo.2014.01.009

9. Bamias A, Efstathiou E, Moulopoulos LA et al (2005) The outcome of elderly patients with advanced urothelial carcinoma after platinum-based combination chemotherapy. Ann Oncol 16:307313. https://doi.org/10.1093/annonc/mdi039

10. Antonelli A, Zamboni S, Palumbo C et al (2018) Prognostic role of delay before radical cystectomy: retrospective analysis of a single-centre cohort with 376 patients. Minerva Urol Nefrol 70:494-500. https://doi.org/10.23736/S0393-2249.18.02995-8

11. Grossman HB, Natale RB, Tangen CM et al (2003) Neoadjuvant chemotherapy plus cystectomy compared with cystectomy alone for locally advanced bladder cancer. N Engl J Med 349:859-866. https://doi.org/10.1056/NEJMoa022148

12. Montironi R, Lopez-Beltran A (2005) The 2004 WHO classification of bladder tumors: a summary and commentary. Int J Surg Pathol 13:143-153. https://doi.org/10.1177/106689690501300203

13. Moschini M, D'Andrea D, Korn S et al (2017) Characteristics and clinical significance of histological variants of bladder cancer. Nat Rev Urol 14:651-668. https://doi.org/10.1038/nrurol.2017.125

14. Greene FL, Page DL, Fleming IDE (2002) Urinary bladder. AJCC cancer staging manual, 6th edn. Springer, New York

15. Shariat SF, Karakiewicz PI, Palapattu GS et al (2006) Outcomes of radical cystectomy for transitional cell carcinoma of the bladder: a contemporary series from the Bladder Cancer Research Consortium. J Urol 176:2414-2422. https://doi.org/10.1016/j. juro.2006.08.004 (discussion 2422)

16. Svatek RS, Shariat SF, Novara G et al (2011) Discrepancy between clinical and pathological stage: external validation of the impact on prognosis in an international radical cystectomy cohort. BJU Int 107:898-904. https://doi.org/10.1111/j.1464410X.2010.09628.x

17. Shariat SF, Palapattu GS, Karakiewicz PI et al (2007) Discrepancy between clinical and pathologic stage: impact on prognosis after radical cystectomy. Eur Urol 51:137-149. https://doi. org/10.1016/j.eururo.2006.05.021 (discussion 149-151)

18. Culp SH, Dickstein RJ, Grossman HB et al (2014) Refining patient selection for neoadjuvant chemotherapy before radical cystectomy. J Urol 191:40-47. https://doi.org/10.1016/j.juro.2013.07.061

19. Moschini M, Soria F, Klatte T et al (2017) Validation of preoperative risk grouping of the selection of patients most likely to benefit from neoadjuvant chemotherapy before radical cystectomy. Clin Genitourin Cancer 15:e267-e273. https://doi.org/10.1016/j. clgc.2016.07.014

20. Abufaraj M, Shariat SF, Foerster B et al (2018) Accuracy and prognostic value of variant histology and lymphovascular invasion at transurethral resection of bladder. World J Urol 36:231-240. https://doi.org/10.1007/s00345-017-2116-3

21. Moschini M, Dell'Oglio P, Luciano' R et al (2017) Incidence and effect of variant histology on oncological outcomes in patients with bladder cancer treated with radical cystectomy. Urol Oncol 35:335-341. https://doi.org/10.1016/j.urolonc.2016.12.006

22. D'Andrea D, Abufaraj M, Susani M et al (2018) Accurate prediction of progression to muscle-invasive disease in patients with pT1G3 bladder cancer: a clinical decision-making tool. Urol Oncol 36:239.e1-239.e7. https://doi.org/10.1016/j.urolo nc. 2018.01 .018

Publisher's Note Springer Nature remains neutral with regard to jurisdictional claims in published maps and institutional affiliations. 